\title{
Ein paar Gedanken zum Thema Autonomie ...
}

\section{Samia Hurst}

Prof. Dr. med., Mitglied der Redaktion, Institut für Bioethik (iEH2), Medizinische Fakultät, Genf

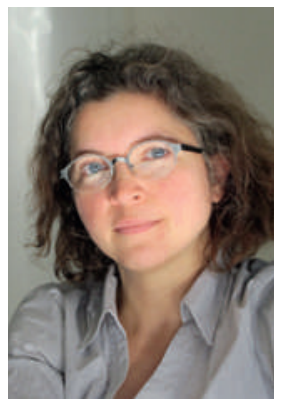

Werte wie Autonomie, Selbstbestimmung und Freiheit sind von grosser Bedeutung. Sie stehen an prominenter Stelle in unseren klinischen Entscheidungen und gesundheitspolitischen Erwägungen. Aus einem philosophischen Blickwinkel betrachtet, sind solche Werte dichter und komplexer Natur. Bei der Erörterung klinischer Fälle oder an Konferenzen werden sie häufig falsch interpretiert. Dabei lassen sich ihre wichtigsten Aspektierungen relativ schnell erfassen. Vielleicht nehmen Sie sich bei einer Tasse Kaffee kurz die Zeit, sich die wesentlichen Punkte zu ihrer Umsetzung nochmals ins Gedächtnis zu rufen.

Erste wichtige Komponente: Unsere Autonomie achten d.h. vor allem, uns gegen Machtmissbrauch schützen. Wir haben ein Grundrecht, nichts gegen unseren Willen zu erdulden. Als Akteure im Vollbesitz unserer Urteilskräfte über uns selbst haben wir das Recht, alle uns betreffenden Interventionen abzulehnen. Zu welchen Bedingungen? Sofern wir verstanden haben, um was es geht und fähig sind, zu bestimmen. Dieses Recht besteht selbst dann, wenn die Intervention eindeutig indiziert - sogar lebensnotwendig ist. Hingegen besteht kein gleicher Rechtsanspruch darauf, eine Intervention zu fordern. Der Schutz der Autonomie des Patienten besteht darin, ihn gegen jede Form von Unterwerfung zu schützen, selbst wenn sie gut gemeint ist. Dies wiederum bedeutet nicht, allen Wünschen des Patienten zu entsprechen. Und vor allem nicht ohne Rücksicht auf allfällige Konsequenzen für Dritte.

\section{Wir haben ein Grundrecht, nichts gegen unseren Willen zu erdulden.}

In der Tat endet unsere Freiheit da, wo die der anderen beginnt. Wenn wir die Autonomie des Patienten achten, heisst dies nicht, dass wir ihm zugestehen, Dritten Verpflichtungen aufzuerlegen. Abhängige ältere Patienten, die gerne zu Hause bleiben möchten, haben beispielsweise das Recht, eine Hospitalisierung abzulehnen. Hingegen dürfen sie nicht verlangen, dass die Mitarbeitenden im Gesundheitsdienst von den betroffenen Angehörigen verlangen, sich zu Hause um sie zu kümmern.

Dieses Beispiel macht auch deutlich, dass die Ziele der
Frage, welche Interventionen nützlich sind und welche nicht, welche fortgesetzt und welche unterbrochen werden, konzentrieren sich die Patienten und ihre Angehörigen globaler auf die jeweiligen Lebensumstände [1]. Zum Ende eines Lebens zählen auch Erwägungen wie Wir müssen unsere Patienten davor schützen, dass über sie bestimmt wird, nicht davor, dass sie sich selbst entscheiden.

die Sorge, nicht leiden zu müssen, das Leben gelebt zu haben, anderen zu helfen, als Individuum respektiert zu werden, gute Beziehungen zu wahren oder sich an einem vertrauten Ort aufhalten zu können. Allzu häufig verwenden wir eine Art Fragenkatalog, in dem es darum geht, die betreffende Intervention als akzeptiert anzukreuzen oder abzulehnen. Dabei sollte eher über die Ziele gesprochen werden, die von der Medizin unterstützt oder möglicherweise behindert werden könnten. Der Ruf nach Autonomie ist kein Aufruf zum Egoismus. Niemand ist gezwungen, wichtige Entscheidungen nur aus Gründen der eigenen Autonomie allein im stillen Kämmerchen treffen zu müssen. Unsere Patienten haben das Recht, ihre Entscheide diskutieren zu können mit wem auch immer sie das möchten. Sie haben sogar das Recht, sich beeinflussen zu lassen. Unsere Entscheide werden häufig gemeinsam mit anderen getroffen. Wir müssen unsere Patienten davor schützen, dass über sie bestimmt wird, nicht davor, dass sie sich selbst entscheiden.

Und nicht zuletzt muss Autonomie auch das Recht auf Irrtum beinhalten. Das Respektieren der Patientenautonomie bedingt auch, dass wir erkennen, dass $\mathrm{Pa}$ tienten - wie wir selbst - Menschen sind, die sich für bestimmte Wege entscheiden. Und dass sie sich dabei irren können - wie wir alle. Natürlich müssen wir ihnen dabei helfen, grobe Fehler zu meiden, soweit dies möglich ist. Letztendlich können wir aber niemanden zwingen. So ist das nun mal im Leben: Ohne das Recht auf Fehler gibt es keine Freiheit ...

\section{Literatur}

1 Steinhauser KE, Clipp EC, McNeilly M, Christakis NA, McIntyre LM, Tulsky JA. In search of a good death: observations of patients, families, and providers. Ann Intern Med. 2000 May 16;132(10):825-32. PubMed PMID: 10819707. 